\title{
Comparison of Simultaneous Dual-Isotope Multipinhole SPECT with Rotational SPECT in a Group of Patients with Coronary Artery Disease
}

\author{
Peter P. Steele, Dennis L. Kirch, and John E. Koss \\ Western Cardiology Associates, Westminster, Colorado
}

\begin{abstract}
A triple-detector, multipinhole SPECT system was optimally configured to perform simultaneous ${ }^{201} \mathrm{TI}$ (stress)/99mTc (rest) myocardial perfusion imaging (MPI) using a protocol that permitted direct diagnostic comparison of this multipinhole SPECT system with conventional rotational SPECT. Methods: Both the rotational and the multipinhole SPECT systems used the same model $\gamma$-detectors. The 2 systems were applied in tandem to study 26 patients with documented coronary status. Visual image evaluation of the MPI together with quantitative analysis using circumferential profile curves (CPC) were used for interpretation of stress/rest myocardial flow differences. A dual-peak attenuation compensation (DPAC) technique was applied to the stress ${ }^{201} \mathrm{TI}$ multipinhole SPECT images by weighted combination of the images from the upper and lower peaks. Results: Detection of myocardial infarction by location and extent correlated closely, and correlation of differential flow changes between stress and rest indicated similar accuracy in terms of location and extent of myocardial blood flow differences as well. In addition, the application of DPAC clarified the multipinhole stress ${ }^{201} \mathrm{Tl}$ images through reduced background and increased statistics and also improved the relative superposition of the normalized CPC, especially for the inferior and more basal reconstructed regions. Conclusion: The prototype 3-detector multipinhole SPECT system achieved diagnostic results comparable to those for rotational SPECT and required only a single image-acquisition session to generate stress/rest MPI and 16-segment poststress gated studies. This reduction in acquisition time significantly improves productivity without compromising diagnostic accuracy. In addition, DPAC is a useful adjunct to the multipinhole SPECT modality because it improves both the visual clarity of the stress images and the stress/rest quantitative comparability.
\end{abstract}

Key Words: myocardial perfusion imaging; SPECT; multipinhole collimation; attenuation correction; circumferential profile curves

J Nucl Med 2008; 49:1080-1089

DOI: 10.2967/jnumed.107.040915

Received Apr. 27, 2007; revision accepted Mar. 27, 2008.

For correspondence or reprints contact: Dennis L. Kirch, Western Cardiology Associates, 8407 Bryant St., Westminster, CO 80031.

E-mail: dlkirch@comcast.net

COPYRIGHT @ 2008 by the Society of Nuclear Medicine, Inc.
$\mathbf{I}_{1}$ patients suspected of having coronary artery disease (CAD), myocardial perfusion imaging (MPI) is generally performed in 2 phases, comparing perfusion images obtained under rest conditions with those obtained separately after stress. Interpretation of the relative perfusion uptake differences between stress and rest is made by visual as well as quantitative evaluation. These rotational SPECT perfusion images are generally useful, but the reported diagnostic accuracy of this methodology has remained unchanged for some time (1-3).

Since the introduction of multipinhole SPECT in 1978 by Vogel et al. (4), several improvements have been made to this methodology. Extension of the multipinhole method to a multidetector SPECT configuration was first proposed by Bizais et al. (5) and Koral et al. (6). Simultaneous stress/rest multipinhole SPECT has been reported (7), which allows the ${ }^{201} \mathrm{Tl}$ (stress) and ${ }^{99 \mathrm{~m}} \mathrm{Tc}$ (rest) MPI to be acquired in a single imaging session. More recently, dual-peak attenuation compensation (DPAC) (8) has been developed to reduce attenuation-induced differences between stress and rest images acquired simultaneously.

In sequentially acquired rotational SPECT projections, temporal changes lead to data inconsistencies between views. In this circumstance, the sequentially acquired rotational projections may see the heart under conditions of changing size, shape, or position from view to view. Attempts to use after-the-fact software corrections have proved difficult. In mathematic terms, such inconsistencies produce ill-conditioned datasets incorporating ambiguities that can potentially generate artifacts in the reconstructed MPI as described by Bacharach (9).

This type of view-to-view inconsistency cannot occur with simultaneous stress/rest multipinhole SPECT because all of the individual views are acquired during the same coincidental time period. It remains true that motion during acquisition can cause blurring or resolution loss in SPECT images, but such motion affects both the stress and the rest multipinhole images equivalently. Therefore, patient motion of any kind is unlikely to produce the false-positive appearance of a stress/rest flow difference in multipinhole SPECT 
images because such data inconsistencies are essentially eliminated.

Additional advantages of multipinhole SPECT MPI include improved patient comfort in terms of arm positioning, reduced incidence of repeated studies due to patient movement, increased patient throughput because only a single image acquisition is required, and a simpler and a more reliable system design with fewer moving parts. The significant gain in sensitivity reported by Funk et al. (10) for multipinhole over rotational SPECT is achieved because the multiplicity of pinhole views uses the available detector surface area more efficiently. The pinhole methodology also allows serial measurement of the left ventricular contractile indices by gated analysis during recovery from stress and high-resolution gated list-mode acquisition using less radioactivity (11). Dynamic flow studies with myocardial tracers such as ${ }^{99 \mathrm{~m}} \mathrm{Tc}$-teboroxime are also feasible.

\section{MATERIALS AND METHODS}

\section{SPECT System Configuration and Modification}

This study compared MPI performed by multipinhole SPECT technique with rotational studies acquired using 2 equivalent multidetector SPECT systems in a group of patients with known CAD. An unmodified camera (Prism 3000XP; Picker) was used to perform conventional rotational SPECT MPI by standard protocol. A second identical system was modified to perform multipinhole SPECT (Fig. 1A) by remounting the 3 detectors at fixed locations, with $67.5^{\circ}$ of angular separation between the middle line-of-sight of each detector. The detectors were remounted with $90^{\circ}$ reorientation from their original position so that the long axis of the detector was parallel to the longitudinal axis of the patient's body (Fig. 1B). This arrangement optimally views the $180^{\circ}$ left anterior oblique region surrounding the cardiac apex.

This multipinhole SPECT design uses 3 detectors $(250 \times$ $375 \mathrm{~mm}$ useful field of view), each equipped with a $2 \times 3$ view multipinhole collimator (6-mm pinhole diameter) to simultaneously project a set of 18 views $(125 \times 125 \mathrm{~mm})$ onto nonoverlapping regions of the detectors. The correction tables were loaded by the manufacturer's recommended procedures, but the detector uniformity correction capability was turned off and a flood image was acquired through each collimator to correct for both detector nonuniformity and pinhole sensitivity variations. Data were acquired in list-mode format.

For rotational SPECT by our imaging protocol, patients were reclined in the supine position for $15 \mathrm{~min}$ before the onset of image acquisition for both the rest and the stress images to allow time for cardiovascular equilibration to occur, thereby reducing both respiratory motion and stabilizing the position and volume of the left ventricle before rotational image acquisition. This procedure reduces the likelihood that the cardiac volume itself will vary during acquisition, but it does not completely eliminate the possibility that other causes of volume change or spurious patient movement can occur (Fig. 2). The simultaneous multipinhole SPECT acquisition protocol, however, allows image acquisition to begin immediately after completion of stress to facilitate the study of left ventricular contraction abnormalities and monitor volume changes during recovery from stress.
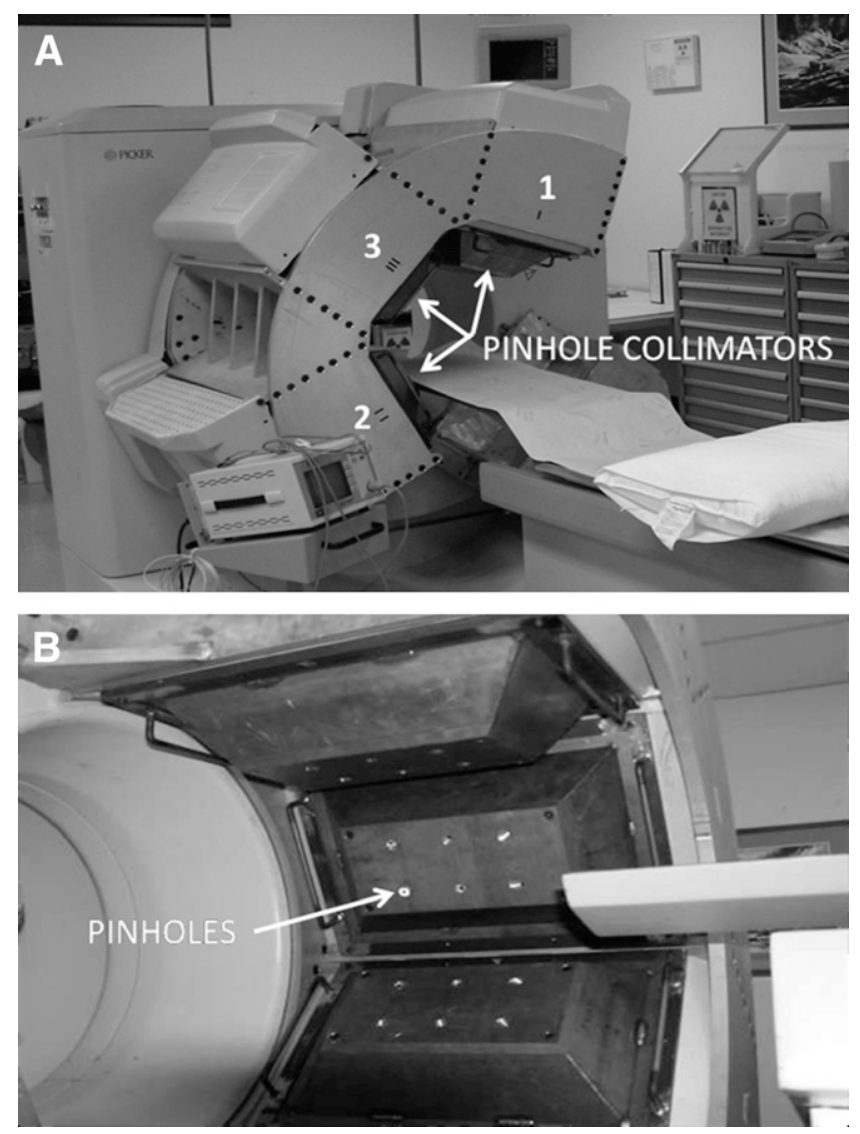

FIGURE 1. (A) Picker Prism 3000XP is shown as modified for multipinhole cardiac SPECT perfusion imaging. System is shown set up for patient to be positioned feet-in so that 3 detectors will surround left anterior oblique position of heart. Detector 3 has been remounted between detectors 1 and 2, and all 3 detectors were remounted $90^{\circ}$ from the original mounting configuration. (B) Close-up of all 3 detectors with pinhole collimators in place.

\section{Patient Inclusion Criterion}

A consecutive series of 26 patients (23 men, 44-73 y) was studied. All had arteriographically defined CAD and had been clinically stable for at least 4 mo. Patients were selected to encompass the range of clinically expressed coronary disease and body builds encountered in practice.

Weights ranged from 57 to $145 \mathrm{~kg}$, and all patients were successfully positioned. Informed consent was reviewed and signed by all patients according to a protocol approved by the Institutional Review Board of Presbyterian/St. Luke's Hospital, Denver, Colorado.

Eighteen patients with prior myocardial infarction were intentionally selected for the location and extent of infarction, which was defined as a region of decreased segmental wall thickening with a history of clinically documented infarction. Previous coronary arteriography and clinical stress testing followed by SPECT MPI were the documentation criteria used to identify patients for inclusion, and the group included those with an extent of CAD from mild to severe.

Coronary arteriography is not a perfect standard of comparison. Often, the degree to which a low-level obstruction (e.g., 50\%$80 \%$ ) might be expected to decrease myocardial flow does not 


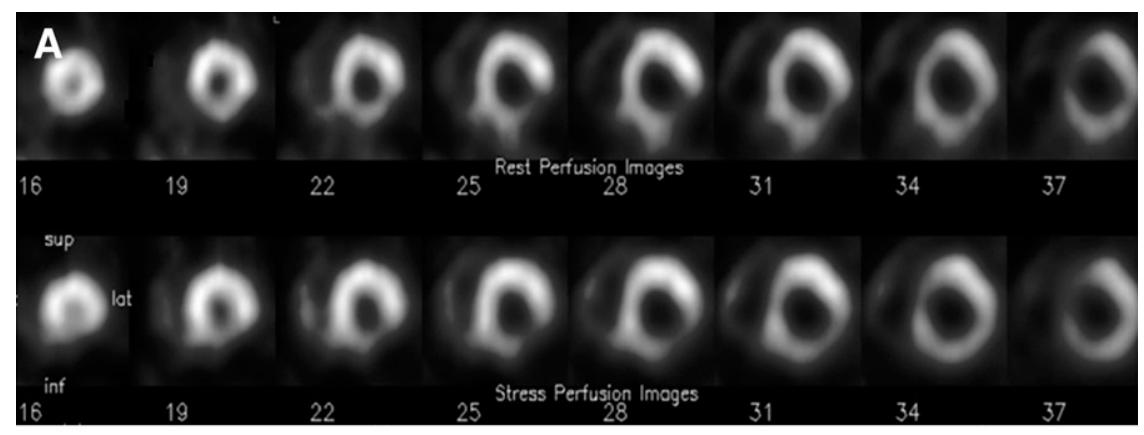

FIGURE 2. Short-axis reconstructions for multipinhole $(A)$ and rotational (B) SPECT acquisitions performed on the same patient showing inferior-lateral defect. Resting images are shown above stress images. Arrows point to artifact caused by patient motion, which occurred during acquisition of resting rotational SPECT images. This type of motion artifact is eliminated by simultaneous multipinhole SPECT technique.

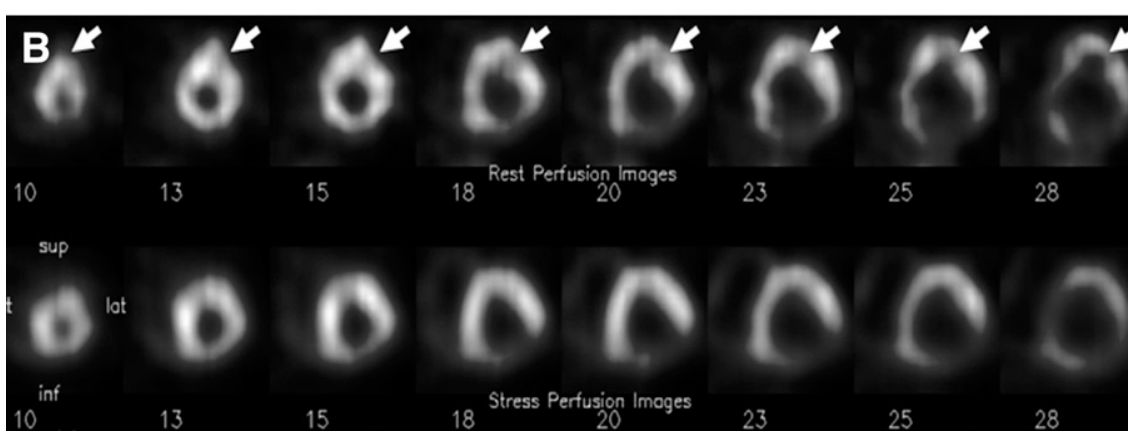

correlate with the observed decrease in stress-induced myocardial blood flow. Patent coronary arteries in areas of infarction, patent bypass grafts, or successful angioplastic intervention do not necessarily support normal myocardial blood flow either.

We avoided the use of rotational SPECT as our reference standard for this study, because the use of rotational SPECT introduces the expectation that images from subsequent modalities should look identical or superior but not in any way different. The mathematic criterion for exact reconstruction of a 3-dimensional distribution function requires a fully 3-dimensional sampling geometry covering $2 \pi$ steradians. This is not physically achievable in the application of SPECT to MPI by either multipinhole or rotational technique. Because multipinhole and rotational SPECT have different sampling geometries, it is expected that the visual appearance of MPI from these 2 modalities should be similar but not identical.

Alternatively, we have used a comparative approach whereby the 2 modalities are applied to a group of patients in which the clinical outcome of SPECT is known in advance and the images are evaluated for quality, as measured by interpretive confidence and correlation with previous coronary arteriographic findings. This approach makes each arteriographic segment available as a separate interpretive test sample, effectively expanding the sample size. It is also assumed that normal native coronary arteries will provide adequate flow after stress testing and, therefore, the corresponding segments should demonstrate normal MPI.

The arteriograms were reviewed for a difference in myocardial blood flow that could possibly occur in a given left ventricular segment with exercise stress. These segments were defined as "tenable segments" in that they represented regions of possible blood flow difference that potentially could result from exercise stress. The clinical definition of a tenable segment required a greater than $50 \%$ decrease in the cross-sectional diameter of the coronary artery supplying that segment. All patients were rightcoronary dominant. That is, both right-coronary- and left-circumflexsupplied segments were present in all patients.
A tenable segment could also include a segment involved in myocardial infarction, independent of whether the coronary artery supplying this segment was patent. This is because changes in left ventricular segmental wall tension in and around areas of infarction can also alter blood flow distribution. A tenable segment was also considered to be one supplied by an artery in which angioplasty or stent placement had occurred (at least 4 mo before inclusion in all cases). This recognizes the potential for various degrees of partial restenosis to occur in arteries after intervention, as was the case for several patients selected for this study. Finally, all segments where a bypass graft had been placed during coronary bypass surgery were considered as tenable, because the designation of "normal flow" cannot be assumed even when graft patency had been previously established.

Four segments of each annular reconstructed short-axis slice were analyzed: anterior, inferior, posterior, and superior. The anterior segment corresponds to the $120^{\circ}$ interventricular septum. Inferior segments are defined as those from the $90^{\circ}$ arc extending toward the posterior segment from the septum and superior segments include the $90^{\circ}$ arc extending from the superior aspect of the septum toward the posterior wall. Posterior segments are those encompassed by the $60^{\circ}$ arc between the superior and the inferior segments. Patient selection allowed up to 4 myocardial segments to show a potential blood flow difference after exercise stress (i.e., tenable segments). It was anticipated that abnormalities in blood flow distribution would not occur in all tenable segments with exercise. The majority of the patients in our group had segments where blood flow maldistribution was a virtual impossibility (i.e., nontenable segments).

\section{Exercise Stress Testing and Imaging Protocols}

To complete both multipinhole and rotational SPECT procedures within the same day for each patient, treadmill exercise testing was undertaken in 2 separate sessions, performed 90-120 min apart to allow for full recovery. Stress testing was performed using the standard Bruce protocol in all cases and was intentionally 
symptom-limited. Only 2 patients had myocardial ischemic signs and symptoms during the exercise test. Exercise duration lasted from 5 to $12 \mathrm{~min}$ before stress injection of ${ }^{201} \mathrm{Tl}$ (thallous chloride; Covidien) for multipinhole SPECT or ${ }^{99 \mathrm{~m} T c}$ (Myoview; GE Healthcare) for rotational SPECT. The peak heart rate achieved during the last minute of exercise for any individual varied by less than $5 \%$ for the paired exercise tests.

After the initial placement of an intravenous line, $222 \mathrm{MBq}$ of ${ }^{99 \mathrm{~m}} \mathrm{Tc}$ were injected with the patient at rest. The patient was then reclined for a 15-min period of equilibration before the acquisition of the resting rotational SPECT image set. The first treadmill exercise test was then performed, with administration of $148 \mathrm{MBq}$ of ${ }^{201} \mathrm{Tl} 1 \mathrm{~min}$ before the end of exercise. Immediately after completion of exercise, the patient was reclined for simultaneous imaging of ${ }^{201} \mathrm{Tl}$ (stress) and ${ }^{99 \mathrm{~m}} \mathrm{Tc}$ (rest) by multipinhole SPECT technique during a single 20-min imaging session, which completed the multipinhole portion of the study. The initial injection of the ${ }^{99 \mathrm{~m}} \mathrm{Tc}$ agent at rest provided data for the resting images for both the rotational and multipinhole SPECT portions of the study.

After an adequate period for recovery, the second graded treadmill exercise test was performed with stress injection of $1,100 \mathrm{MBq}$ of ${ }^{99 \mathrm{~m}} \mathrm{Tc}$. The patient was then reclined in a supine position for acquisition of the final rotational SPECT stress image acquisition after equilibration.

\section{Data Acquisition, Reconstruction, and Analysis}

During multipinhole SPECT image acquisition, the combined stress/rest data were streamed into a list-mode file that was then reformatted offline $(1.78 \mathrm{~mm} / \mathrm{pixel})$ to correct for scatter, cross talk, and nonuniformity. DPAC was then applied to the stress ${ }^{201} \mathrm{Tl}$ images. All rotational SPECT studies were acquired in the standard step-and-shoot mode using low-energy, high-resolution collimation at $40 \mathrm{~s} / \mathrm{step}$ in $6^{\circ}$ increments and framed into $64 \times 64$ pixel matrices $(5.34 \mathrm{~mm} / \mathrm{pixel})$. Both modalities were reconstructed using 12 iterations of a maximum-likelihood expectation maximization algorithm with filtered backprojection as the initial estimate. This acquisition protocol supports reconstruction of the gated SPECT images into 16-frame animations per R-to-R interval, with comparable statistical quality for both modalities. Scatter and attenuation correction were not applied to the rotational SPECT studies.

The stress/rest MPI differences were quantified by application of CPC to 8 equally spaced short-axis annular images between the apex and the base of the heart for both the rotational and the multipinhole studies. Middle-of-the-wall, maximum-value tracking in $6^{\circ}$ increments around each short-axis slice was performed using an automated program. Each stress and rest CPC was initially normalized to $100 \%$ at the maximum value. On review, if this normalization process resulted in CPC showing the resting curve falling significantly below the stress curves $(>5 \%$ over any contiguous $30^{\circ}$ segment), then the point of normalization was relocated to eliminate this anomalous region. We consider this circumstance (termed reverse redistribution) to be diagnostically erroneous and constrain the resting curve to be substantially equal to or above the stress curve over every $30^{\circ}$ segmental portion of the myocardium. This same normalization process was applied to scale the intensity of the stress and rest MPI before visual evaluation so that quantitative differences in the CPC would be consistent with the visual appearance in corresponding regions.

When CPC and MPI are normalized by simply setting the maximum uptake value of each individual curve to $100 \%$, criss-crossing patterns or resting defects are frequently observed for which no satisfactory clinical explanation exists. In this circumstance, our approach to normalization applies a constraint that prevents the resting curve from tracking significantly below the stress curve and also eliminates the appearance of defects in the resting images, which are not seen in the stress images. Curves and images normalized in this manner support interpretive results that are better correlated with the clinical findings. The reader for this study used the MPI and CPC exclusively for interpretation. The gated images were not included in the interpretive process for this study because of the more subjective aspect inherent to their inclusion.

\section{Attenuation Compensation Methodology}

Over the energy range of ${ }^{201} \mathrm{Tl}$ that is useful for MPI, there are 2 peaks (a lower peak at $69-83 \mathrm{keV}$ and a higher peak at $167.5 \mathrm{keV}$ ) that contain radionuclide emissions useful for estimation of the MPI attenuation characteristics. These 2 peaks conveniently bracket the ${ }^{99 \mathrm{~m} T c}$ peak. By the DPAC approach $(8)$, the $\gamma$-emissions attributed to the lower and upper peaks of ${ }^{201} \mathrm{Tl}$ are additively combined after scatter correction. A cardiac insert in an anthropomorphic torso phantom (Data Spectrum) was used to determine empirically that equal weighting was optimum. This was evaluated for visual and quantitative equivalence between ${ }^{201} \mathrm{Tl}$ and ${ }^{99 \mathrm{~m}} \mathrm{Tc}$ multipinhole SPECT images. This process generates composite stress ${ }^{201} \mathrm{Tl}$ multipinhole images that have visual and quantitative characteristics that more nearly correspond to those of the resting ${ }^{99 \mathrm{~m} T c}$ multipinhole images. We did not apply any attenuation correction methodology to the resting ${ }^{99 \mathrm{~m}} \mathrm{Tc}$ multipinhole SPECT MPI before reconstruction.

\section{RESULTS}

Visual Interpretation of Location and Extent of Infarction

In this study, 18 of the 26 patients had a prior myocardial infarction. As noted earlier, patients were selected to include all potential locations for infarction and to cover a range of infarction sizes corresponding to left ventricular ejection fractions ranging from 0.25 to 0.63 . In all patients with infarction, the location was consistently identified in both the multipinhole and rotational SPECT images (e.g., an apical-anterior infarction in one modality was not an anterior-superior or anterior-inferior infarction in the other imaging modality and so on). Visual inspection of infarct size was comparable between the 2 methodologies, although as previously reported (12-14), the appearance of defects in the ${ }^{201} \mathrm{Tl}$ multipinhole SPECT images was slightly larger.

\section{Visual Interpretation of Flow Differences}

The comparison of paired stress/rest MPI studies in patients with prior infarction and in those without infarction resulted in multipinhole and rotational SPECT images that are visually similar but not identical, as previously discussed. This is the major factor contributing to the visual differences in the multipinhole and rotational short-axis images shown in our results (Fig. 2). However, these differences did not significantly affect clinical interpretation of the studies included here because interpretation depends on the magnitude and extent of relative differences visually observed between the stress and MPI (Fig. 3) and quantified 


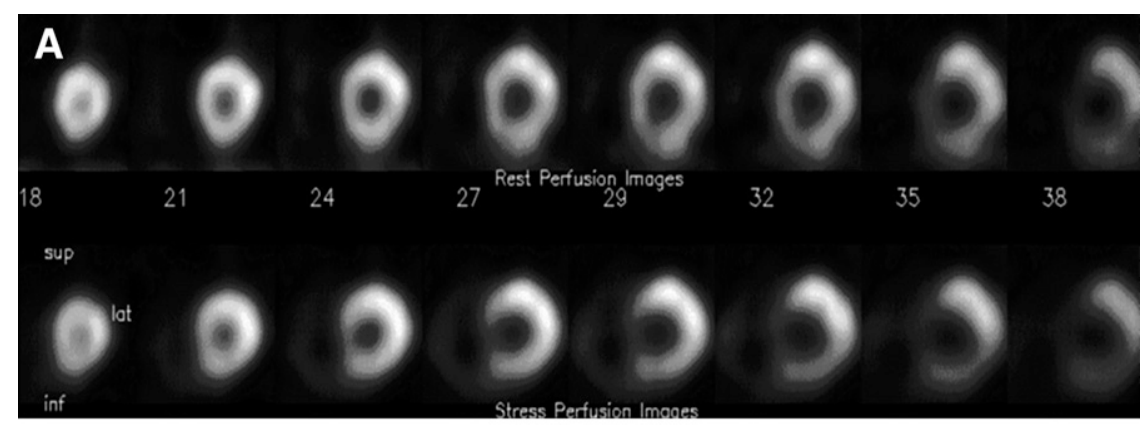

FIGURE 3. Short-axis reconstructions for multipinhole (A) and rotational (B) SPECT acquisitions performed on patient with anterior flow difference. Resting images are shown above stress images. Images from 2 modalities are visually and quantatively similar. (See curves in Fig. 4.) Same areas of anterior flow difference are identified in both sets of images, but images in A show larger stress defect than rotational SPECT results in $B$.

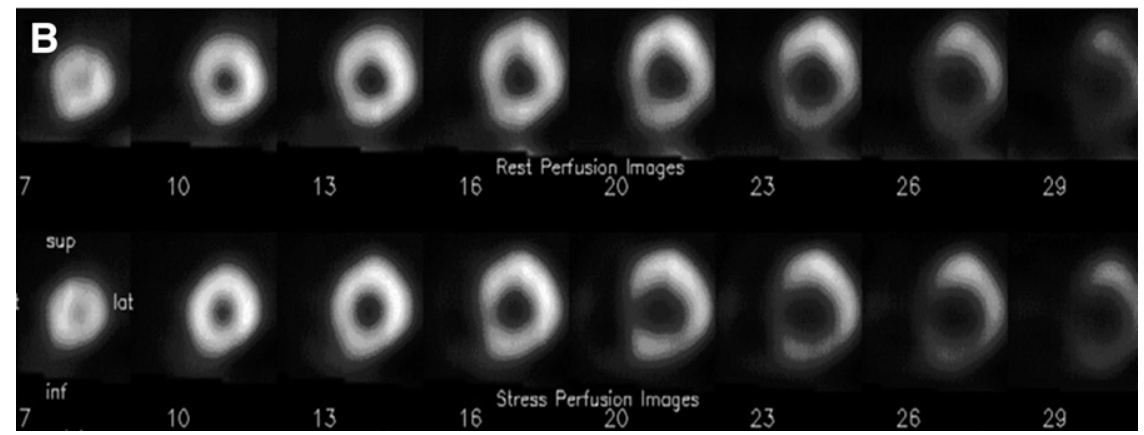

by CPC analysis of normalized stress/rest image sets (Figs. 4-6). The clinical interpreter felt that the multipinhole images were generally easier to interpret and provided greater certainty in grading abnormalities, although we recognize that this is a subjective impression.

\section{CPC Analysis}

CPC quantification provides an objective means to support precise visual image evaluation. A comparative example of the short-axis MPI and CPC on the same patient is shown in Figures 3 and 4, respectively. For the tenable segments, general agreement is demonstrated by the multipinhole and rotational SPECT perfusion images. Furthermore, results from CPC analysis of myocardial blood flow differences in the 18 patients with documented infarction correlated well with the images produced by both the multipinhole and the rotational methodologies. Thirty-seven segments (of 72 possible) were tenable. In rotational SPECT studies, 18 of $37(49 \%)$ showed a flow difference. For the multipinhole curves, 19 of 37 (51\%) demonstrated a significant difference $(>10 \%)$.

Although most of the blood flow differences in these patients were in segments that also contained an infarct, no relationship between patency of the infarct-related artery and the presence or extent of flow differences existed. The presence of an infarct-related scar poses another nonobstructive mechanism of maldistribution of blood flow arising from altered myocardial wall tension. Positive findings in left bundle branch block represent a similar observation. In this small group of patients, analysis of the product of heart rate times the blood pressure at the end of exercise did not correlate well with quantitative measurements of flow differences. However, extending this concept to a larger group of patients might prove useful. Vasodilator stress MPI might be fruitful in correlating flow differences in areas of infarction for patients who otherwise are difficult to evaluate clinically.

In this study group, 8 patients have not had infarction. Of 32 possible segments 20 were considered to have a potential flow difference with exercise stress (tenable segments). With rotational SPECT, 9 of the 20 (45\%) segments demonstrated flow differences, whereas 10 of the $20(50 \%)$ tenable segments had a flow difference with multipinhole SPECT. Again, the quantitative precision obtained with circumferential profile analysis was essential for demonstration of flow differences in segments supplied by arteries with less severe blockages. An example of this is demonstrated visually in the multipinhole images compared with those obtained with rotational SPECT in a patient with left anterior descending obstruction (Fig. 5).

Rotational SPECT studies demonstrated flow differences in 5 of the 26 patients (19\%) who had flow differences in angiographically defined nontenable segments. These differences (Fig. 6) occur most often in apical-superior segments and are typically small differences that an experienced observer might safely ignore. However, these small changes occurred in a segment that is often supplied by the diagonal branch of the left anterior descending coronary artery, which challenges its exclusion. None of the multipinhole SPECT perfusion images had flow differences in nontenable segments.

\section{Attenuation Compensation}

Our dual-peak approach to attenuation compensation, compared with the multipinhole images without attenuation compensation, resulted in images with less noise, enhanced contrast (lower background), and clinically consistent profile curves in 8 patients (31\%) (Fig. 7). However, no instances in 


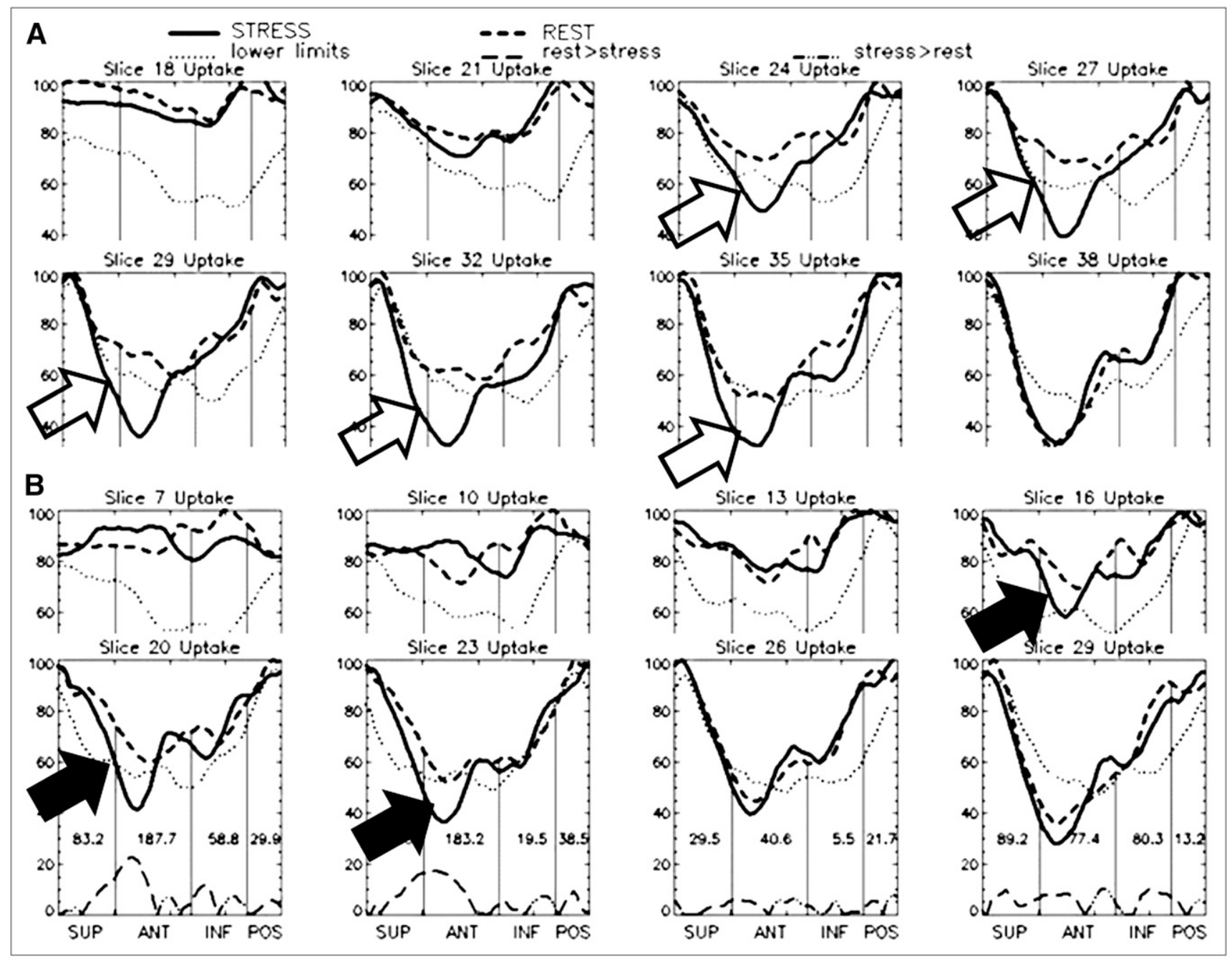

FIGURE 4. CPC of short-axis images shown in Figure 3 for multipinhole (A) and rotational (B) SPECT reconstructions. Same anterior regions of flow difference are identified in both sets of curves, but multipinhole analysis shows greater flow differentiation (open arrows) than rotational analysis (closed arrows). SUP = superior; ANT = anterior; INF = inferior; POS = posterior.

which a diagnostic difference was revealed after application of DPAC existed. The clinical interpreter felt that diagnostic certainty was increased by the application of DPAC.

This approach uses intrinsic information available from the multiple energy peaks of ${ }^{201} \mathrm{Tl}$ as acquired during simultaneous stress/rest imaging. Therefore, no additional radiation exposure is imposed, and no alignment issues in registering the attenuation information with the images themselves exist. As would be expected, the inferior- and posterior-basal segments benefited most from application of DPAC (Fig. 7), because they are deeper within the thorax.

We term the process described here attenuation compensation, rather than attenuation correction, because we have not advanced its use to compute an attenuation map or attempted absolute quantification of myocardial blood flow. Our DPAC approach should be compared with attenuation maps generated by the more rigorous $\mathrm{CT}$ method because the possibility exists, with proper validation and correlation, that this approach could be extended to achieve absolute blood flow quantification. The results presented here represent the interpretation of the perfusion images and CPC by a single reader. The gated images were not used for the interpretive results reported here.

\section{Cumulative Review of Comparative Results}

Supplemental data, including the MPI, CPC, gated images, and clinical correlation for all 26 patients reported on in this article, are available online only at http://jnm. snmjournals.org. The fundamental premise for clinical interpretation of MPI is based on the concept that the resting portion of the study serves as the reference standard for gauging the normalcy of the stress portion. This approach is most accurate when systematic errors are minimized, reducing the erroneous appearance of flow differences in the CPC. Systematic errors are most often related to spurious patient movement or changes in the myocardial configuration during image acquisition, which tend to create both positive and negative differences between the stress/ rest CPC (i.e., criss-crossing of the normalized CPC). In the nontenable segments, the multipinhole SPECT MPI 


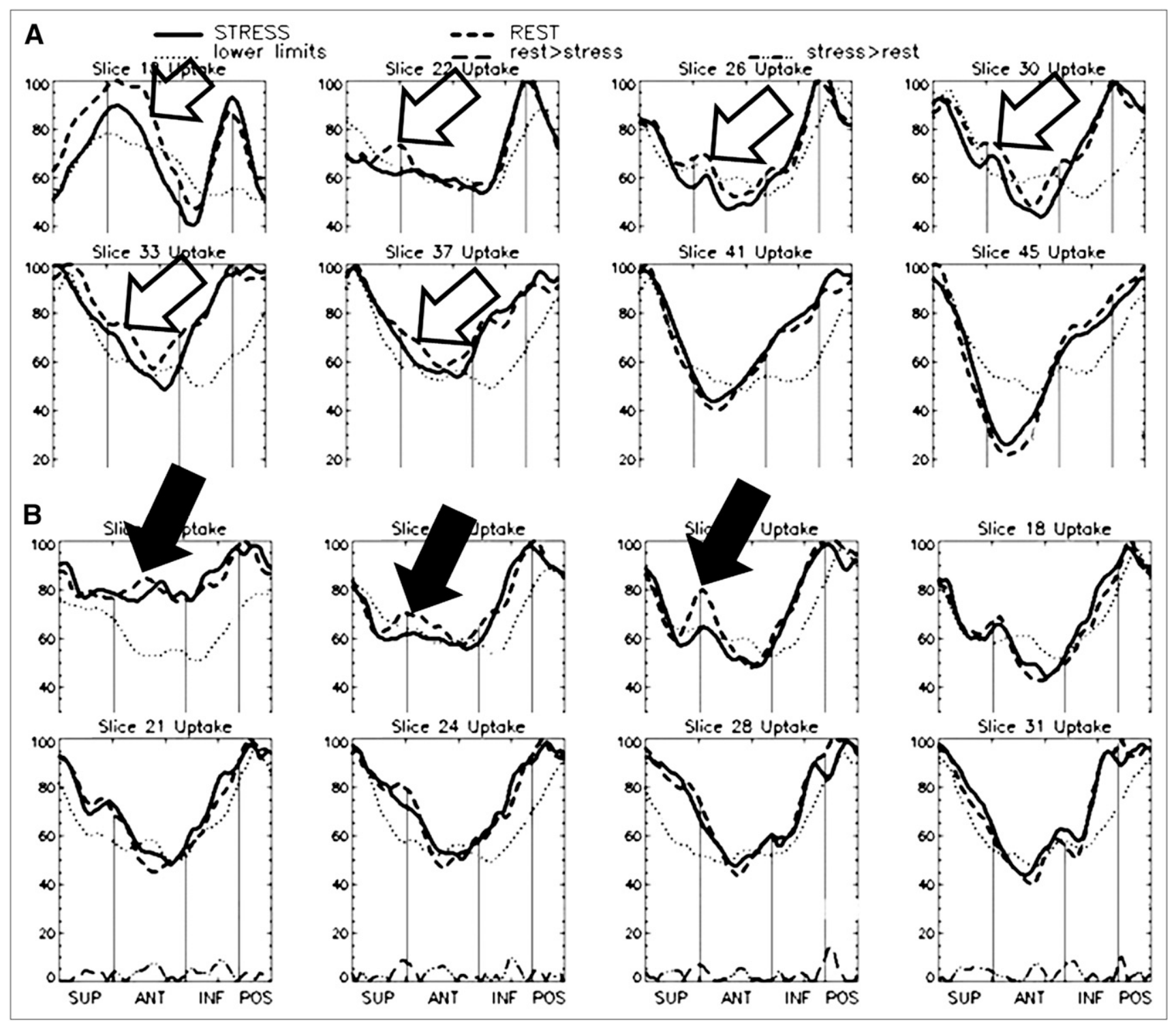

FIGURE 5. CPC of short-axis slices from multipinhole $(A)$ and rotational $(B)$ SPECT acquisitions on patient with anterior infarct. Low-level flow differences as seen in region of infarct in multipinhole study (open arrows) are also demonstrated, but to lesser extent, in rotational study (closed arrows). SUP = superior; ANT = anterior; INF = inferior; POS = posterior.

demonstrated CPC differences in the range of $\pm 5 \%$ whereas the rotational SPECT MPI tend to show $\pm 10 \%$ differences. On this basis, the simultaneously imaged multipinhole SPECT results are considered less subject to systematic errors and provide increased interpretive certainty.

\section{DISCUSSION}

In the group of patients studied here, we have demonstrated that multipinhole SPECT MPI compared with rotational SPECT achieved similar diagnostic accuracy for detection of the location and size of myocardial infarction and measurement of the location and extent of myocardial blood flow differences. By multipinhole technique, 29 segments of a possible 56 tenable segments indicated a flow difference, whereas 27 of these segments showed a flow difference with rotational SPECT. The patients in our study were selected to include many subtle flow differences in the tenable segments, so it is difficult to know with absolute certainty whether multipinhole is more sensitive than rotational imaging. In this study group, however, sensitivity was not compromised by the simultaneous ${ }^{99 \mathrm{~m} T \mathrm{Tc} / 201} \mathrm{Tl}$ multipinhole SPECT protocol.

A primary benefit of the multipinhole SPECT approach was borne out by the absence of the type of motion-induced defects to which rotational SPECT is subject, as seen in Figure 2. If such artifactual segments are excluded, little diagnostic difference between multipinhole and rotational SPECT techniques in this group of patients is demonstrated. The use of a 15-min preimage equilibration period for rotational SPECT image acquisition is an important qualifier 


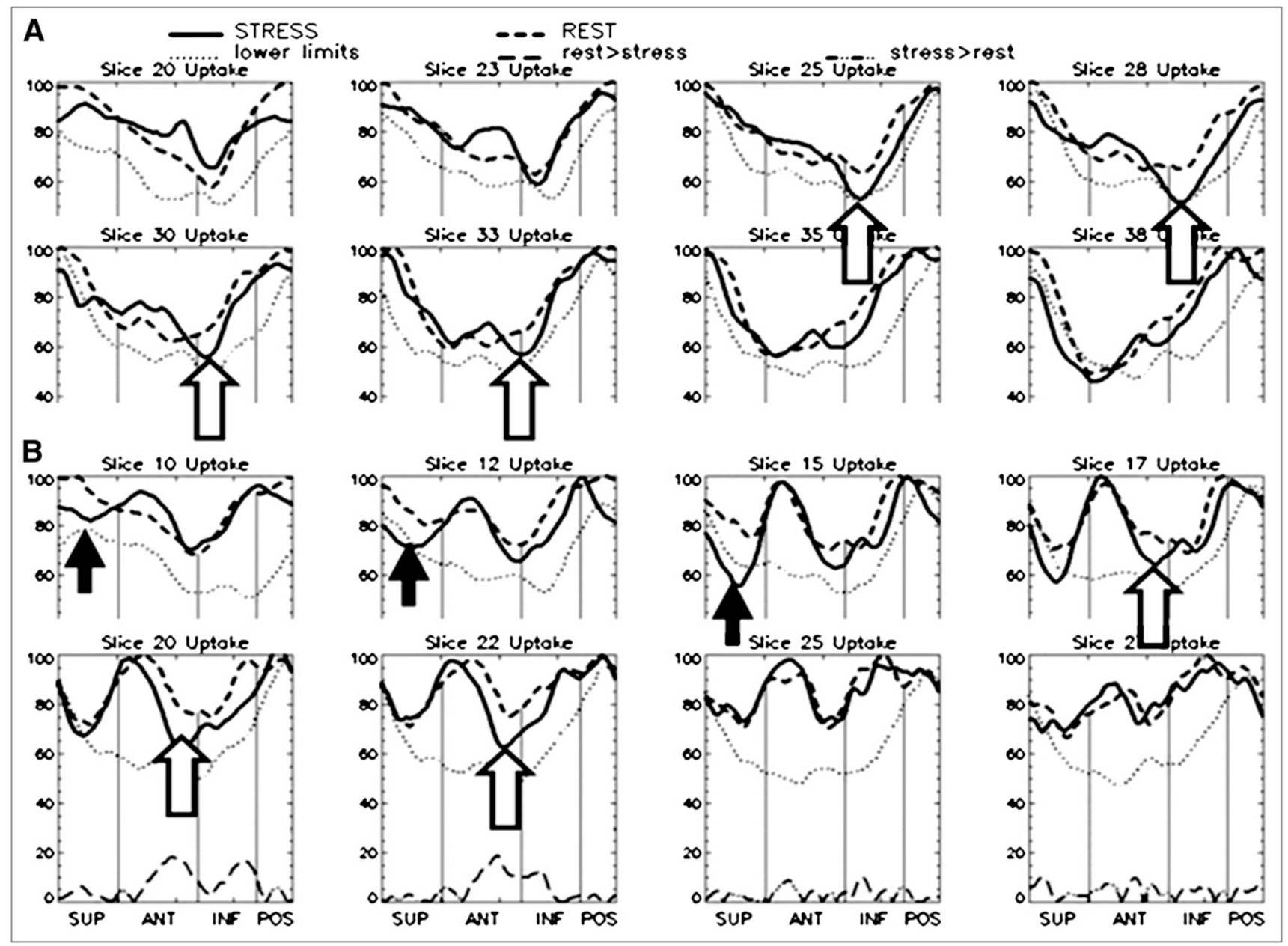

FIGURE 6. CPC of short-axis slices from multipinhole (A) and rotational (B) SPECT acquisitions on patient with inferior defect. Curves for both $A$ and $B$ analyses show inferior flow differences (open arrows), which are consistent with patient's history. Rotational study also shows flow differences in superior apical region (solid arrows), which are inconsistent with location of any known obstructions. SUP = superior; ANT = anterior; INF = inferior; POS = posterior.

to this finding. As has been reported elsewhere, starting rotational SPECT too soon after exercise can lead to falsepositive findings in rotational SPECT due to upward creep (15) or cardiac volume changes.

Variability in cardiac position due to respiration during the rotational imaging period may also be a factor contributing to the artifacts in rotational images. The predominantly apical-superior location of the artifacts suggests this possibility. Rotational images were analyzed both with and without the commercially supplied motion-correction program, but this additional analysis did not successfully eliminate these artifacts. The higher detection sensitivity achieved by multipinhole SPECT lends itself to a motioncorrection scheme that is capable of accommodating respirational motion. In preliminary studies, correction for respiratory motion achieved resolution improvement in reconstructed multipinhole SPECT images (16).

DPAC improved image quality when applied to the multipinhole. In no instance was a diagnostic difference apparent between uncompensated versus DPAC multipinhole im- ages. DPAC images demonstrated lower background and the CPC were less noisy in about one third of the stress images for the patients in this study group. In addition, attenuation compensation is considered to reduce systematic error and enhance interpretive diagnostic certainty and is easily incorporated into routine imaging preprocessing when list-mode data are available. The level of systematic error observed in clinical imaging has been reduced from $\pm 10 \%$ to $\pm 5 \%$ by the introduction of DPAC.

Brown et al. (17) first suggested the use of the high peak of ${ }^{201} \mathrm{Tl}$ to characterize attenuation effects. However, their approach was based on the use of rotational SPECT with limited statistical content, which allowed only identification of specific patients whose studies would benefit from more rigorous attenuation correction such as might be available from dual-modality SPECT/CT. The sensitivity of the rotational SPECT camera systems used for comparative purposes here and in the work of Brown et al. (17) are very similar; therefore, our attenuation-compensation approach could not be applied to the rotational MPI reported here. 


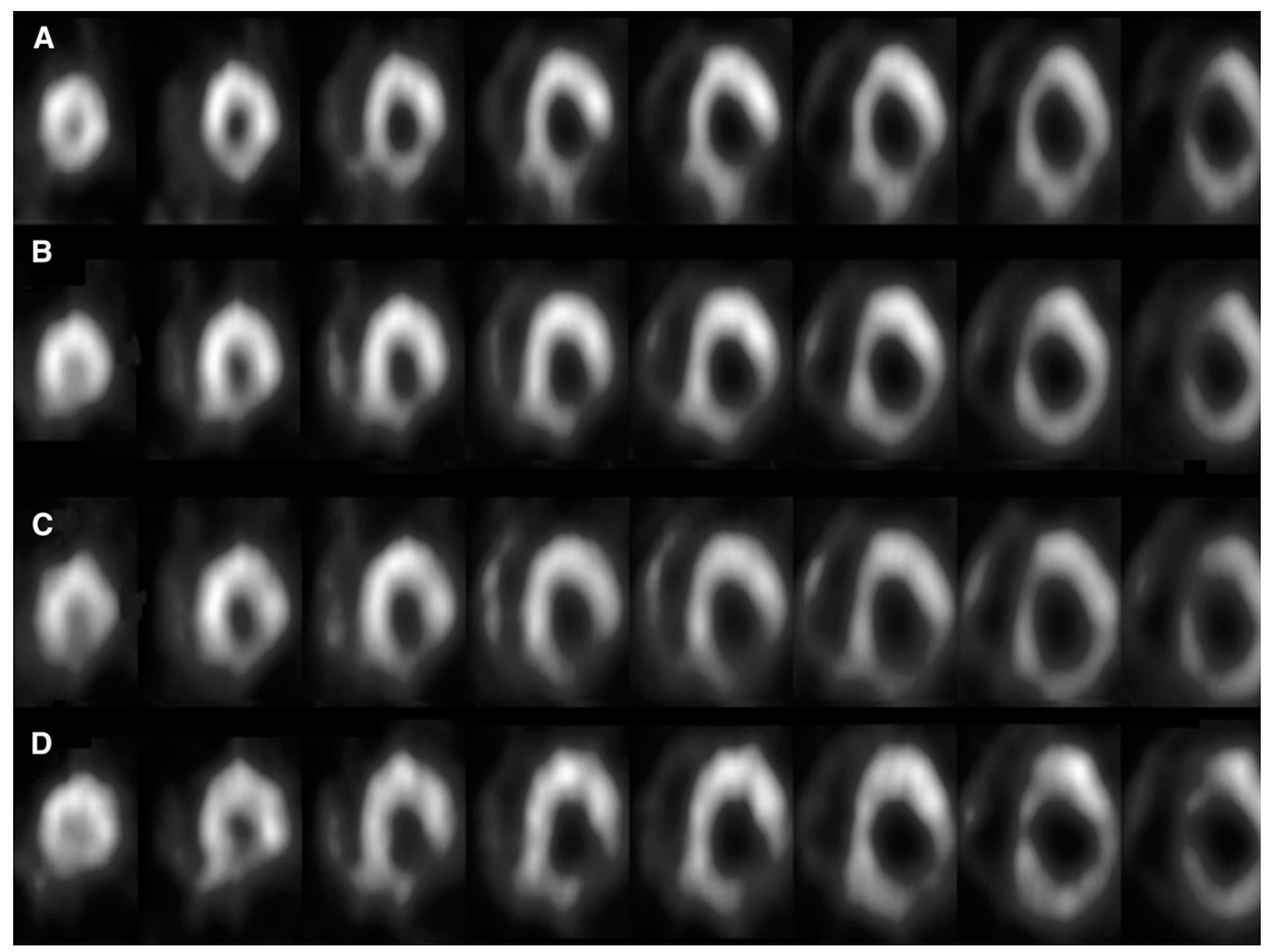

FIGURE 7. Short-axis reconstructions from multipinhole SPECT MPI demonstrating DPAC. (A) Resting images using 99mTc tetrofosmin. (B) Simultaneously acquired ${ }^{201} \mathrm{TI}$ stress reconstructions combining both peaks of $201 \mathrm{TI}$. (C and D) Separate reconstructions for lower peak (C) and upper peak (D) of ${ }^{201} \mathrm{TI}$. Attenuation-compensated stress images in B visually compare better to resting images in $A$ than stress images from either of 2 individual thallium peaks shown in $C$ and $D$.

The ${ }^{99 m}$ Tc activity injected (222 MBq for the simultaneous multipinhole SPECT protocol) was chosen to provide adequate resting count statistics without overwhelming the stress counts present in the region of the lower ${ }^{201} \mathrm{Tl}$ peak (148 $\mathrm{MBq}$ ) with Compton scatter. At these injected activity levels, the quality of the multipinhole studies was maintained while reducing the imaging time by half. This is a direct result of the increased detection sensitivity of multipinhole SPECT, which provides additional diagnostic information such as left ventricular volume, ejection fraction, contractile indices, segmental wall motion, and thickening during recovery from stress. This result points to the future prospects that multipinhole SPECT can be applied to other clinical applications such as myocardial viability, as well as true dynamic SPECT studies of tracer kinetics leading to the ability to make absolute measurements of myocardial blood flow.

Finally, even though significantly less ${ }^{99 \mathrm{~m}} \mathrm{Tc}$ activity was needed for simultaneous multipinhole SPECT, equivalent diagnostic accuracy was obtained with only a single combined stress/rest imaging session. Moreover, the fact that patient, staff, and facility procedure times are reduced for multipinhole SPECT without compromising diagnostic outcome is also important.

\section{CONCLUSION}

Multipinhole SPECT as described in this article, using a stationary multidetector system, increases detection sensitivity and reduces systematic errors associated with MPI. The improved statistical content of these images facilitates simultaneous dual-isotope stress/rest MPI, DPAC of the stress images, more precise stress/rest quantification of flow differences, and reduced incidence of motion-induced artifactual features in the reconstructed images.

In the patient group studied here, multipinhole SPECT images are comparable in diagnostic accuracy with those produced by the rotational SPECT technique at reduced cost, improved patient comfort, and an expenditure of time and effort that is approximately one half that of rotational SPECT. The elimination of mechanical motion by the 
multipinhole approach reduces the complexity associated with both the manufacture and the maintenance of rotational dedicated cardiac SPECT systems.

The multipinhole approach also supports other diagnostic capabilities that are difficult to perform by rotational acquisition. This is accomplished in conjunction with quantification using CPC, which facilitates both precise quantification of perfusion flow differences and accurate measurement of gated cardiac performance. The ability to form all images simultaneously also represents a significant step toward the ability to quantify dynamic tracer uptake and washout characteristics leading to absolute flow measurements.

\section{ACKNOWLEDGMENTS}

We thank Drs. Bruce Hasegawa and Tobias Funk of the University of California, San Francisco, for many helpful suggestions regarding approaches to the multipinhole, multidetector design. Dr. J. Keenan Brown of Mindways Software, Inc., provided useful guidance on methods for reconstruction of images generated from limited angular sampling geometry. Gary Jarkewicz of Philips Healthcare provided essential information for modification of the Picker Prism 3000XP, and Al Blount provided significant contributions to the assembly, calibration, and technical support of the modified system. We also thank Jeffery Nobles, Michael Adams, and Louise Renoux for their capable technical assistance.

\section{REFERENCES}

1. Van Train KF, Garcia EV, Maddahi J, et al. Multicenter trial validation for quantitative analysis of same-day rest-stress technetium-99m sestamibi myocardial tomograms. J Nucl Med. 1994;35:609-618.

2. Patterson R, Horowitz S, Eisner R. Comparison of modalities to diagnose coronary artery disease. Semin Nucl Med. 1994;24:286-310.
3. Des Prez RD, Shaw LJ, Gillespe RL, et al. Cost-effectiveness of myocardial perfusion imaging: a summary of the currently available literature. $\mathrm{J} \mathrm{Nucl}$ Cardiol. 2005;12:750-759.

4. Vogel RA, Kirch DL, Lefree M, et al. A new method of multiplanar emission tomography using a seven pinhole collimator and an Anger scintillation camera. J Nucl Med. 1978;19:648-654.

5. Bizais Y, Bennett GW, Brill AB, Rowe RW, Zubal IG. Dual seven pinhole tomography. IEEE Trans Nucl Sci. 1983;30:703-706.

6. Koral KF, Clinthorne NH, Rogers WL, Keyes JW Jr. Feasibility of sharpening limited-angle tomography by including an orthogonal set of projections. Nucl Instrum Methods. 1982;193:223-227.

7. Kirch DL, Koss JE, Steele PP, Johnson TK. Performance and clinical trials of a multi-pinhole nonrotational cardiac SPECT system [abstract]. J Nucl Med. 2000;41(suppl):177P.

8. Kirch DL, Koss JE, Steele PP. Dual-peak attenuation compensation (DPAC) using the upper and lower Tl-201 peaks during simultaneous stress/rest multipinhole SPECT (MP-SPECT) for myocardial perfusion imaging (MPI) [abstract]. J Nucl Med. 2007;48(suppl 2):99P.

9. Bacharach SL. Why does SPECT work? J Nucl Cardiol. 2006;13:313-315.

10. Funk T, Kirch DL, Koss JE, et al. A novel approach to multi-pinhole SPECT for myocardial perfusion imaging. J Nucl Med. 2006;47:595-602.

11. Koss JE, Steele PP, Kirch DL. Increased spatial and temporal resolution of gated myocardial perfusion images (GMPI) achieved by multi-pinhole SPECT (MP-SPECT) [abstract]. J Nucl Med. 2007;48(suppl 2):420P.

12. Matsunari I, Fujino S, Taki J, et al. Comparison of defect size between thallium201 and technetium-99m tetrofosmin myocardial single-photon emission computed tomography in patients with single-vessel coronary artery disease. Am J Cardiol. 1996;77:350-354.

13. Zaret BL, Rigo P, Wackers FJ, et al. Tetrofosmin International Trial Study Group. Myocardial perfusion imaging with ${ }^{99 \mathrm{~m}} \mathrm{Tc}$ tetrofosmin: comparison to ${ }^{201} \mathrm{Tl}$ imaging and coronary angiography in a phase III multicenter trial. Circulation. 1995;91:313-319.

14. Glover DK, Ruiz M, Yang JY, et al. Myocardial ${ }^{99 \mathrm{~m}}$ Tc-tetrofosmin uptake during adenosine-induced vasodilatation with either a critical or mild coronary stenosis: comparison with ${ }^{201} \mathrm{Tl}$ and regional myocardial blood flow. Circulation. 1997;96:2332-2338.

15. Friedman J, Van Train K, Maddahi J, et al. "Upward creep" of the heart: a frequent source of false-positive reversible defects during thallium-201 stressredistribution SPECT. J Nucl Med. 1989;30:1718-1722.

16. Kirch DL, Koss JE, Steele PP. Motion correction (MC) of muliti-pinhole SPECT (MP-SPECT) myocardial perfusion imaging (MPI) on breath-by-breath basis improves accuracy in measurement of LV function [abstract]. $\mathrm{J} \mathrm{Nucl} \mathrm{Med}$. 2007;48(suppl 2):42P.

17. Brown JK, Tang HR, Hattner RS, et al. Intrinsic dual-energy processing of myocardial perfusion images. J Nucl Med. 2000;41:1287-1297. 